\title{
Fractional Brownian Motion Delayed by Tempered and Inverse Tempered Stable Subordinators
}

\author{
A. Kumar' ${ }^{1}$ J. Gajda² - A. Wyłomańska² . \\ R. Połoczański ${ }^{2}$
}

Received: 2 June 2017 /

Accepted: 8 June 2018 / Published online: 19 June 2018

(C) The Author(s) 2018

\begin{abstract}
In recent years subordinated processes have been widely considered in the literature. These processes not only have wide applications but also have interesting theoretical properties. In this paper we consider fractional Brownian motion (FBM) time-changed by two processes, tempered stable and inverse tempered stable. We present main properties of the subordinated FBM such as long range dependence and associated fractional partial differential equations for the probability density functions. Moreover, we present how to simulate both subordinated processes.
\end{abstract}

Keywords Subordination - Tempered stable process - Inverse tempered stable process · Fractional Brownian motion · Simulation

Mathematics Subject Classification (2010) $60 \mathrm{G} 20 \cdot 60 \mathrm{G} 10 \cdot 35 \mathrm{R} 11$

\section{Introduction}

In the recent years subordinated processes have been widely considered in the literature. In general, a subordinated process is constructed by taking superposition of two independent stochastic systems. The evolution of time in the external process is replaced by a non-decreasing stochastic process, called subordinator. Due to this fact, subodinators can

J. Gajda

janusz.gajda@pwr.edu.pl

\section{A. Kumar}

aruns.iitb@gmail.com

1 Department of Mathematics, Indian Institute of Technology Ropar, Nangal Road, Rupnagar, Punjab 140001, India

2 Faculty of Pure and Applied Mathematics, Hugo Steinhaus Center, Wrocław University of Science and Technology, Wybrzeże Wyspiańskiego 27, 50-370 Wrocław, Poland 
be treated as the "operational time". The resulting subordinated processes very often retain important properties of the external process, however certain characteristics might change. The idea of subordination was introduced in 1949 by Bochner $(1949,1955)$. The theory of subordinated processes is also explored in details in Sato (1999). The subordinated processes have found many interesting applications, for example in finance (Clark 1973), physics (Nezhadhaghighi et al. 2011), ecology (Scher et al. 2002) and hydrology (Doukhan et al. 2003).

In this paper we consider the fractional Brownian motion (FBM) time-changed by two processes, tempered stable and inverse tempered stable. The FBM is a classical long-range dependent and self-similar process used for description of so-called anomalous diffusion phenomena. The tempered stable Lévy process is strongly related to stable Lévy process, i.e. process with stationary independent increments with stable distribution. The stable processes are very often considered in the literature (Fristedt 1977). However, due to the infinite second moments (except the Gaussian case), their applications require usage of advanced mathematical methods. The tempered stable processes possess many properties of stable systems however their second moments are finite and classical methods of analysis can be applied here. The inverse processes in recent years have been considered by many authors (Wyłomańska 2013). The interest of those processes is related to the fact that time-changed processes delayed by inverse subordinators are strongly related to the continuous time random walk model, the second classical model used for description of anomalous diffusion phenomena (Stanislavsky et al. 2014). The most often used subordinator is the stable one, however in the literature one can find examples of others (Magdziarz 2009), see also Barndorff-Nielsen et al. (2001).

In this paper we consider first the tempered stable and inverse tempered stable processes. We present their main properties such as moments asymptotic behaviors and associated fractional partial differential equations for their probability density functions. Next, we consider the FBM time-changed by two mentioned systems, i.e. tempered stable and inverse tempered stable. We compare main characteristics of time-changed FBM with the equivalent characteristics of classical FBM. The main attention is paid to long range dependence property of new time-change processes and the associated fractional partial differential equations for probability density functions. We also present the procedure of simulating both subordinated processes.

\subsection{Tempered Stable Subordinator}

Before we introduce the tempered stable subordinator (TSS) it is worth mentioning the class of stable processes. Stable distributions belong to an important class of probability distributions. These distributions possess heavy tails and have many intriguing mathematical properties. Stable distributions are characterized by four parameters: stability index $\alpha \in(0,2]$, skewness parameter $\beta \in[-1,1]$, scale parameter $\sigma>0$ and shift parameter $\mu \in R$. The formal definition of stable distribution one can find in Samorodnitsky and Taqqu (1994). We only mention, stable distributions are infinitely divisible and hence also provide a rich class of Lévy processes also called stable (or $\alpha$-stable) Lévy processes. These processes are self-similar i.e. for an $\alpha$-stable process $D_{\alpha}(t)$, it follows $\left\{D_{\alpha}(c t), t \geq 0\right\} \stackrel{\mathcal{L}}{=}$ $\left\{c^{1 / \alpha} D_{\alpha}(t), t \geq 0\right\}$ for $c>0$, where $\underline{\mathcal{L}}$ denotes equality of finite dimensional distributions. Despite having interesting properties the applications of these processes are limited due to non-finiteness of second order moments (except the Gaussian case). To overcome 
these limitations tempered stable processes are introduced (Rosiński 2007). Tempered stable processes are infinitely divisible, have exponentially decaying tail probabilities and have all moments finite. As mentioned in Section 1, subordinator is a process that can replace the time of other stochastic process and is a non-decreasing and non-negative Lévy process. Thus the tempered stable subordinator (TSS) is the non-decreasing and non-negative tempered stable Lévy process. Similarly, the $\alpha$-stable subordinator is a non-decreasing and non-negative $\alpha$-stable Lévy process.

Let $f_{\alpha}(x, t)$ be the density function of an $\alpha$-stable subordinator $D_{\alpha}(t)$. In this case the stability parameter $\alpha \in(0,1)$, the skewness $\beta=1$ and shift $\mu=0$. In our analysis we assume the scale parameter $\sigma=1$. The integral form of the probability density function (PDF) is given by Kumar and Vellaisamy (2015)

$$
f_{\alpha}(x, t)=\frac{1}{\pi} \int_{0}^{\infty} e^{-x y} e^{-t y^{\alpha} \cos \alpha \pi} \sin \left(t y^{\alpha} \sin \alpha \pi\right) d y .
$$

Let $\mathcal{L}_{x \rightarrow u}(g(x, t))=\tilde{g}(u, t)$ be the Laplace transform (LT) of $g$ with respect to space variable $x$. Then

$$
\tilde{f}_{\alpha}(u, t)=\mathbb{E}\left(e^{-u D_{\alpha}(t)}\right)=\int_{0}^{\infty} e^{-u x} f_{\alpha}(x, t) d x=e^{-t u^{\alpha}} .
$$

Let $S_{\lambda, \alpha}(t)$ be the TSS with index $\alpha \in(0,1)$ and tempering parameter $\lambda>0$. Note that TSS are obtained by exponential tempering in the distributions of stable processes (Rosiński 2007). TSS $S_{\lambda, \alpha}(t)$ has density function (Rosiński 2007)

$$
f_{\lambda, \alpha}(x, t)=e^{-\lambda x+\lambda^{\alpha} t} f_{\alpha}(x, t), \quad \lambda>0, \alpha \in(0,1) .
$$

Using Eqs. 2 and 3, the LT of $f_{\lambda, \alpha}(x, t)$ is given by

$$
\tilde{f}_{\lambda, \alpha}(u, t)=\int_{0}^{\infty} e^{-u x} f_{\lambda, \alpha}(x, t) d x=e^{-t\left((u+\lambda)^{\alpha}-\lambda^{\alpha}\right)} .
$$

It is worthwhile to mention that for $\lambda=0$, TSS reduces to $\alpha$-stable subordinator and for $\alpha=1 / 2, \alpha$-stable subodiantor is also called $1 / 2$-stable subordinator (or Lévy subordinator) (see e.g. Applebaum 2009). Next, the asymptotic forms of the moments of TSS $S_{\lambda, \alpha}(t)$ are presented, which will be used further in establishing the LRD property of the FBM timechanged by the TSS. First we provide a formula to calculate the fractional order moments for a positive random variable by using its LT. Next we use this formula to calculate the fractional order moments of TSS $S_{\lambda, \alpha}(t)$. Note that for a positive random variable $X$ with Laplace transform $\tilde{f}(u)$, it follows

$$
\begin{aligned}
& \int_{0}^{\infty} \frac{d^{n}}{d u^{n}}[\tilde{f}(u)] u^{p-1} d u \\
& =\int_{0}^{\infty} \frac{d^{n}}{d u^{n}}\left[\mathbb{E} e^{-u X}\right] u^{p-1} d u \\
& =\mathbb{E}\left[\int_{0}^{\infty} \frac{d^{n}}{d u^{n}}\left[e^{-u X}\right] u^{p-1} d u\right] \quad \text { (by Fubini theorem) } \\
& =(-1)^{n} \mathbb{E}\left[X^{n} \int_{0}^{\infty} e^{-u X} u^{p-1} d u\right] \\
& =(-1)^{n} \Gamma(p) \mathbb{E}\left(X^{n-p}\right),
\end{aligned}
$$


where $\Gamma(p)$ is the gamma function. Using Eq. 5, the $q$-th order moment of $X$, where $q \in(n-1, n)$ for some integer $n$, is given by

$$
\mathbb{E}\left(X^{q}\right)=\frac{(-1)^{n}}{\Gamma(n-q)} \int_{0}^{\infty} \frac{d^{n}}{d u^{n}}[\tilde{f}(u)] u^{n-q-1} d u .
$$

Note that by using the above formula, we can explicitly calculate the fractional order moments of TSS $S_{\lambda, \alpha}(t)$ which for given $t$ is a positive random variable. Thus for $q>0$, it leads to the following result.

Proposition 1 For $q>0$, the asymptotic behavior of $q$-th order moments of $\operatorname{TSS} S_{\lambda, \alpha}(t)$ is given by

$$
\mathbb{E}\left(S_{\lambda, \alpha}(t)\right)^{q} \sim\left(\alpha \lambda^{\alpha-1} t\right)^{q}, \text { as } t \rightarrow \infty
$$

Proof We prove this result in two parts. For fractional $q$ and integer $q$ different approaches are used. For $0<q<1$, using Eq. 6, it follows

$$
\begin{aligned}
\mathbb{E}\left(S_{\lambda, \alpha}(t)\right)^{q} & =\frac{(-1)}{\Gamma(1-q)} \int_{0}^{\infty} \frac{d}{d u}\left[e^{-t\left((u+\lambda)^{\alpha}-\lambda^{\alpha}\right)}\right] u^{-q} d u \\
& =\frac{\alpha t e^{t \lambda^{\alpha}}}{\Gamma(1-q)} \int_{0}^{\infty} u^{-q}(u+\lambda)^{\alpha-1} e^{-t(u+\lambda)^{\alpha}} d u .
\end{aligned}
$$

By choosing $f(u)=(u+\lambda)^{\alpha}$ and $g(u)=(u+\lambda)^{\alpha-1} u^{-q}$, it follows

$$
\begin{aligned}
f(u) & =\lambda^{\alpha}+\alpha \lambda^{\alpha-1} u+\frac{\alpha(\alpha-1) \lambda^{\alpha-2}}{2 !} u^{2}+\cdots \\
& =f(0)+\sum_{k=0}^{\infty} a_{k} u^{k+\beta},
\end{aligned}
$$

where $f(0)=\lambda^{\alpha}, a_{0}=\alpha \lambda^{\alpha-1}, a_{1}=\alpha(\alpha-1) \lambda^{\alpha-2} / 2$ ! and $\beta=1$. Further,

$$
\begin{aligned}
g(u) & =\lambda^{\alpha-1}+(\alpha-1) \lambda^{\alpha-2} u^{1-q}+\frac{(\alpha-1)(\alpha-2) \lambda^{\alpha-3}}{2 !} u^{2-q}+\cdots \\
& =\sum_{k=0}^{\infty} b_{k} u^{k+\gamma-1},
\end{aligned}
$$

where $b_{0}=\lambda^{\alpha-1}, b_{1}=(\alpha-1) \lambda^{\alpha-2}$ and $\gamma=1-q$. Using Laplace-Erdelyi theorem (see Appendix A), we have

$$
\mathbb{E}\left(S_{\lambda, \alpha}(t)\right)^{q} \sim \frac{\alpha t}{\Gamma(1-q)} \sum_{k=0}^{\infty} \Gamma(k+1-q) \frac{c_{k}}{t^{k+1-q}},
$$

where $c_{k}$ in terms of coefficients $a_{k}$ and $b_{k}$ is given by

$$
c_{k}=\frac{1}{a_{0}^{(k+\gamma) / \beta}} \sum_{j=0}^{k} b_{k-j} \sum_{i=0}^{j}\left(\begin{array}{c}
-\frac{k+\gamma}{\beta} \\
i
\end{array}\right) \frac{1}{a_{0}^{i}} \hat{B}_{j, i}\left(a_{1}, a_{2}, \cdots, a_{j-i+1}\right),
$$

and $\hat{B}_{j, i}$ are the partial (or incomplete) ordinary Bell polynomials (Andrews 1998) (see Appendix A). For large $t$ the dominating term is the first one in the series given in Eq. 8, which leads to,

where $c_{0}=\alpha^{q-1} \lambda^{q(\alpha-1)}$.

$$
\mathbb{E}\left(S_{\lambda, \alpha}(t)\right)^{q} \sim c_{0} \alpha t^{q}
$$


Similarly, for a general $q \in(n-1, n)$ we have

$$
\begin{aligned}
\mathbb{E}\left(S_{\lambda, \alpha}(t)\right)^{q} & =\frac{(-1)^{n}}{\Gamma(n-q)} \int_{0}^{\infty} \frac{d^{n}}{d u^{n}}\left[e^{-t\left((u+\lambda)^{\alpha}-\lambda^{\alpha}\right)}\right] u^{n-1-q} d u \\
& \sim \frac{\alpha^{n} t^{n} e^{t \lambda^{\alpha}}}{\Gamma(n-q)} \int_{0}^{\infty} u^{n-1-q}(u+\lambda)^{n(\alpha-1)} e^{-t(u+\lambda)^{\alpha}} d u \\
& \sim \frac{\alpha^{n} t^{n}}{\Gamma(n-q)} \sum_{k=0}^{\infty} \Gamma(k+n-q) \frac{d_{n}}{t^{k+n-q}} \\
& \sim \alpha^{n} d_{0} t^{q},
\end{aligned}
$$

where $d_{n}$ has similar form as in Eq. 9 and $d_{0}=\left(\alpha \lambda^{\alpha-1}\right)^{q-n} \lambda^{n(\alpha-1)}$. Next for integer $n$ the $n$-th order moment of the $S_{\lambda, \alpha}(t)$ is established. Denoting $\psi(u)=\left((-u+\lambda)^{\alpha}-\lambda^{\alpha}\right) t$, the $n$-th order cumulant $\kappa_{n}=\left.\frac{d^{n}}{d u^{n}} \psi(u)\right|_{u=0}$ is given by

$$
\kappa_{n}=(-1)^{n-1} \alpha(\alpha-1)(\alpha-2) \cdots(\alpha-n+1) \lambda^{\alpha-n} t .
$$

In particular, for $S_{\lambda, \alpha}(t)$, we have $\kappa_{1}=\mathbb{E}\left(S_{\lambda, \alpha}(t)\right)=\alpha \lambda^{\alpha-1} t$ and $\kappa_{2}=\operatorname{Var}\left(S_{\lambda, \alpha}(t)\right)=$ $\alpha(1-\alpha) \lambda^{\alpha-2} t$. Further, the $n$-th order moment satisfies (Rota and Shen 2000; Smith 1995)

$$
\begin{aligned}
\mathbb{E}\left(S_{\lambda, \alpha}(t)\right)^{n} & =\sum_{k=1}^{n} B_{n, k}\left(\kappa_{1}, \kappa_{2}, \cdots, \kappa_{n-k+1}\right) \\
& \sim\left(\kappa_{1}\right)^{n}=\left(\alpha \lambda^{\alpha-1} t\right)^{n}, \text { as } t \rightarrow \infty,
\end{aligned}
$$

where $B_{n, k}$ are partial (or incomplete) exponential Bell polynomials (see Appendix A).

Next, we provide the fractional partial differential equation (FPDE) governed by the density of the TSS and leads to the following result.

Proposition 2 The density $f_{\lambda, \alpha}(x, t)$ satisfies

$$
\frac{\partial}{\partial x} f_{\lambda, \alpha}(x, t)=\left(\lambda^{\alpha}-\frac{\partial}{\partial t}\right)^{1 / \alpha} f_{\lambda, \alpha}(x, t)-\lambda f_{\lambda, \alpha}(x, t)-\delta(x) f_{\lambda, \alpha}(0, t) .
$$

Proof Note that

$$
\begin{aligned}
\left(\lambda^{\alpha}-\frac{\partial}{\partial t}\right)^{1 / \alpha} \tilde{f}_{\lambda, \alpha}(u, t) & =\sum_{j=0}^{\infty}\left(\begin{array}{c}
1 / \alpha \\
j
\end{array}\right)(-1)^{j}\left(\lambda^{\alpha}\right)^{1 / \alpha-j} \frac{\partial^{j}}{\partial t^{j}} \tilde{f}_{\lambda, \alpha}(u, t) \\
& =\sum_{j=0}^{\infty}\left(\begin{array}{c}
1 / \alpha \\
j
\end{array}\right)\left(\lambda^{\alpha}\right)^{1 / \alpha-j}\left((u+\lambda)^{\alpha}-\lambda^{\alpha}\right)^{j} \tilde{f}_{\lambda, \alpha}(u, t) \\
& =\lambda \tilde{f}_{\lambda, \alpha}(u, t) \sum_{j=0}^{\infty}\left(\begin{array}{c}
1 / \alpha \\
j
\end{array}\right)\left(\left(1+\frac{u}{\lambda}\right)^{\alpha}-1\right)^{j} \\
& =(\lambda+u) \tilde{f}_{\lambda, \alpha}(u, t) \\
& =\left(u \tilde{f}_{\lambda, \alpha}(u, t)-f_{\lambda, \alpha}(0, t)\right)+f_{\lambda, \alpha}(0, t)+\lambda \tilde{f}_{\lambda, \alpha}(u, t) .
\end{aligned}
$$

The result follows by application of invert LT to both sides. 


\subsection{Inverse Tempered Stable Subordinator}

Let $E_{\lambda, \alpha}(t)$ be the right continuous inverse of tempered stable process $S_{\lambda, \alpha}(t)$, defined by

$$
E_{\lambda, \alpha}(t)=\inf \left\{y>0: S_{\lambda, \alpha}(y)>t\right\}, \quad t \geq 0 .
$$

The process $E_{\lambda, \alpha}(t)$ is called inverse tempered stable (ITS) subordinator. A driftless subordinator $D(t)$ with Lévy measure $\pi_{D}$ and density function $f$ has the Lévy-Khinchin representation (Bertoin 1996)

$$
\int_{0}^{\infty} e^{-u x} f_{D(t)}(x) d x=e^{-t \Psi_{D}(u)}
$$

where

$$
\Psi_{D}(u)=\int_{0}^{\infty}\left(1-e^{-u y}\right) \pi_{D}(d y), \quad u>0
$$

is called the Laplace exponent. The Lévy measure density corresponding to a tempered stable subordinator is given by Cont and Tankov (2003)

$$
\pi_{S_{\lambda}, \alpha}(u)=\frac{c e^{-\lambda x}}{x^{\alpha+1}}, c>0, u>0,
$$

which satisfies the condition $\int_{0}^{\infty} \pi_{S_{\lambda}, \alpha}(u) d u=\infty$. The sample paths of $S_{\lambda, \alpha}(t)(\alpha \in$ $(0,1), \lambda>0)$ are strictly increasing with jumps and hence the sample paths of $E_{\lambda, \alpha}(t)$ are almost surely continuous and are constant over the intervals where $S_{\lambda, \alpha}(t)$ have jumps. Let $\mathcal{L}_{t \rightarrow s}(g(x, t))=\bar{g}(x, s)$ be the Laplace transform (LT) of $g$ with respect to time variable $t$. Using Meerschaert and Scheffler (2008), the LT of the density $h_{\lambda, \alpha}(x, t)$ of $E_{\lambda, \alpha}(t)$ with respect to the time variable $t$ is given by

$$
\bar{h}_{\lambda, \alpha}(x, s)=\frac{1}{s}\left((s+\lambda)^{\alpha}-\lambda^{\alpha}\right) e^{-x\left((s+\lambda)^{\alpha}-\lambda^{\alpha}\right)} .
$$

The density function $h_{\lambda, \alpha}(x, t)$ can be obtained by inverting the LT in Eq. 16 using the complex-inversion formula with the help of a key-hole contour. Alternatively,

$$
\begin{aligned}
\mathbb{P}\left(E_{\lambda, \alpha}(t) \leq x\right) & =\mathbb{P}\left(S_{\lambda, \alpha}(x) \geq t\right) \\
& =\int_{t}^{\infty} f_{\lambda, \alpha}(y, x) d y \\
& =\int_{t}^{\infty} e^{-\lambda y+\lambda^{\alpha} x} f_{\alpha}(y, x) d y \\
& =\frac{1}{\pi} \int_{y=t}^{\infty} \int_{w=0}^{\infty} e^{-(\lambda+w) y} e^{\lambda^{\alpha} x-x w^{\alpha} \cos \alpha \pi} \sin \left(x w^{\alpha} \sin \alpha \pi\right) d w d y \\
& =\frac{e^{-\lambda t}}{\pi} \int_{0}^{\infty} \frac{e^{-w t}}{\lambda+w} e^{x\left(\lambda^{\alpha}-w^{\alpha} \cos \alpha \pi\right)} \sin \left(x w^{\alpha} \sin \alpha \pi\right) d w .
\end{aligned}
$$

Taking derivatives in both sides with respect to $x$ yields the density function of $E_{\lambda, \alpha}(t)$, that is

$$
\begin{aligned}
h_{\lambda, \alpha}(x, t)= & \frac{e^{-\lambda t+\lambda^{\alpha} x}}{\pi} \int_{0}^{\infty} \frac{e^{-t w-x w^{\alpha} \cos \alpha \pi}}{\lambda+w} \\
& \times\left[\lambda^{\alpha} \sin \left(x w^{\alpha} \sin \alpha \pi\right)+w^{\alpha} \sin \left(\alpha \pi-x w^{\alpha} \sin \alpha \pi\right)\right] d w .
\end{aligned}
$$

Using the identity from Abramowitz and Stegun (1972), namely

$$
\int_{0}^{\infty} e^{-t u^{2}} \cos (a u) d u=\frac{\sqrt{\pi}}{2 \sqrt{t}} e^{-a^{2} /(4 t)}
$$


and by substituting $\lambda=0$ and $\alpha=1 / 2$ in Eq. 17, it follows

$$
h_{0,1 / 2}(x, t)=\sqrt{\frac{2}{\pi t}} e^{-x^{2} /(2 t)} .
$$

Observe that the density of inverse 1/2-stable subordinator given in Eq. 19 is same as density of $|B(t)|$, where $B(t)$ is standard Brownian motion.

Next we obtain the FPDE governed by the PDF of ITS subordinator. The RiemannLiouville (RL) fractional derivative of order $\beta$ for a function $f$ is given by Gorenflo and Mainardi (1997)

$$
\frac{\partial^{\beta}}{\partial t^{\beta}} f(t)= \begin{cases}\frac{d^{m}}{d t^{m}}\left[\frac{1}{\Gamma(m-\beta)} \int_{0}^{t} \frac{f(\tau)}{(t-\tau)^{\beta+1-m}} d \tau\right], & m-1<\beta<m, \\ \frac{d^{m}}{d t^{m}} f(t), & \beta=m .\end{cases}
$$

First we find the governing equation of the density of $E_{\lambda, \alpha}(t)$. In order to do so, we need to introduce the Laplace transform of shifted fractional RL derivative given by Beghin (2015)

$$
\mathcal{L}_{t}\left\{\left(c+\frac{\partial}{\partial t}\right)^{v} f(x, t)\right\}=(c+s)^{v} \mathcal{L}_{t}\{f(x, t)\}-(c+s)^{\nu-1} f(x, 0), \quad s>0 .
$$

In the further analysis we will use the generalized Mittag-Leffler function, therefore we introduce it here. The generalized Mittag-Leffler function, introduced by Prabhakar (1971), is defined by

$$
M_{p, q}^{r}(z)=\sum_{n=0}^{\infty} \frac{(r)_{n}}{\Gamma(p n+q)} \frac{z^{n}}{n !}
$$

where $p, q, r \in \mathbb{C}$ with $\mathcal{R}(q)>0$ and $(r)_{n}=\frac{\Gamma(r+n)}{\Gamma(r)}$ is Pochhammer symbol. When $r=1$, it reduces to Mittag-Leffler function. Further,

$$
M_{p, q}^{r}(0)=\frac{(r)_{0}}{\Gamma(q)}=\frac{1}{\Gamma(q)} .
$$

We will use the following result in next proposition. We remind that the function $F(s)=$ $\frac{s^{p r-q}}{\left(s^{p}+a\right)^{r}}$ has the inverse LT (Monje et al. 2010)

$$
\mathcal{L}^{-1}[F(s)]=t^{q-1} M_{p, q}^{r}\left(-a t^{p}\right) .
$$

Proposition 3 The density function $h_{\lambda, \alpha}(x, t)$ satisfies

$$
\frac{\partial}{\partial x} h_{\lambda, \alpha}(x, t)=-\left(\lambda+\frac{\partial}{\partial t}\right)^{\alpha} h_{\lambda, \alpha}(x, t)+\lambda^{\alpha} h_{\lambda, \alpha}(x, t)-t^{-\alpha} M_{1,1-\alpha}^{1-\alpha}(-\lambda t) \delta(x)
$$

with $h_{\lambda, \alpha}(x, 0)=\delta(x)$.

Proof Using Eq. 16, note that

$$
\bar{h}_{\lambda, \alpha}(x, s)=\frac{1}{s}\left((s+\lambda)^{\alpha}-\lambda^{\alpha}\right) e^{-x\left((s+\lambda)^{\alpha}-\lambda^{\alpha}\right)},
$$

which implies

$$
\begin{aligned}
\frac{\partial}{\partial x} \bar{h}_{\lambda, \alpha}(x, s)= & -\left[(s+\lambda)^{\alpha}-\lambda^{\alpha}\right] \bar{h}_{\lambda, \alpha}(x, s) \\
= & -\left[(s+\lambda)^{\alpha} \bar{h}_{\lambda, \alpha}(x, s)-(s+\lambda)^{\alpha-1} h_{\lambda, \alpha}(x, 0)\right]-(s+\lambda)^{\alpha-1} h_{\lambda, \alpha}(x, 0) \\
& +\lambda^{\alpha} \bar{h}_{\lambda, \alpha}(x, s) .
\end{aligned}
$$


Taking the inverse LT on both sides and using Eq. 21, we get

$$
\frac{\partial}{\partial x} h_{\lambda, \alpha}(x, t)=-\left(\lambda+\frac{\partial}{\partial t}\right)^{\alpha} h_{\lambda, \alpha}(x, t)-\mathcal{L}^{-1}\left[(s+\lambda)^{\alpha-1}\right] \delta(x)+\lambda^{\alpha} h_{\lambda, \alpha}(x, t) .
$$

By taking $p=1, q=1-\alpha, r=1-\alpha$ and $a=\lambda$, we have from Eq. 24

$$
\mathcal{L}^{-1}\left[\frac{s^{1(1-\alpha)-(1-\alpha)}}{\left(s^{1}+\lambda\right)^{1-\alpha}}\right]=\mathcal{L}^{-1}\left[\frac{1}{(s+\lambda)^{1-\alpha}}\right]=t^{-\alpha} M_{1,1-\alpha}^{1-\alpha}(-\lambda t) .
$$

The result follows by using Eqs. 26 and 27.

Taking $\lambda=0$ in Eq. 25 and using Eq. 23, it follows

$$
\frac{\partial}{\partial x} h_{0, \alpha}(x, t)=-\frac{\partial^{\alpha}}{\partial t^{\alpha}} h_{0, \alpha}(x, t)-\frac{t^{-\alpha}}{\Gamma(1-\alpha)} \delta(x),
$$

with $h_{0, \alpha}(x, 0)=\delta(x)$, which is the equation satisfied by the density of inverse $\alpha$-stable subordiantor. Note that this result complements the result obtained in Meerschaert and Straka (2013). Next we complement the result presented in Proposition 3 which involves a first order partial derivative in space variable with an analogs time derivative equation for the density of the ITS subordinator.

Proposition 4 The density function $h_{\lambda, \alpha}(x, t)$ satisfies the following equation

$$
\frac{\partial}{\partial t} h_{\lambda, \alpha}(x, t)=\left(\lambda^{\alpha}-\frac{\partial}{\partial x}\right)^{1 / \alpha} h_{\lambda, \alpha}(x, t)-\lambda h_{\lambda, \alpha}(x, t)+\delta(t) \delta(x) .
$$

with $h_{\lambda, \alpha}(x, 0)=\delta(x)$.

Proof We will prove this by the Laplace transform technique

$$
\begin{aligned}
\left(\lambda^{\alpha}-\frac{\partial}{\partial x}\right)^{1 / \alpha} \bar{h}_{\lambda, \alpha}(x, s) & =\sum_{j=0}^{\infty}\left(\begin{array}{c}
1 / \alpha \\
j
\end{array}\right)\left(\lambda^{\alpha}\right)^{1 / \alpha-j}(-1)^{j} \frac{1}{s}\left((s+\lambda)^{\alpha}-\lambda^{\alpha}\right) \frac{d^{j}}{d x^{j}} e^{-x\left((s+\lambda)^{\alpha}-\lambda^{\alpha}\right)} \\
& =\frac{1}{s}\left((s+\lambda)^{\alpha}-\lambda^{\alpha}\right) e^{-x\left((s+\lambda)^{\alpha}-\lambda^{\alpha}\right)} \sum_{j=0}^{\infty}\left(\begin{array}{c}
1 / \alpha \\
j
\end{array}\right)\left(\lambda^{\alpha}\right)^{1 / \alpha-j}\left((s+\lambda)^{\alpha}-\lambda^{\alpha}\right)^{j} \\
& =\frac{1}{s}\left((s+\lambda)^{\alpha}-\lambda^{\alpha}\right) e^{-x\left((s+\lambda)^{\alpha}-\lambda^{\alpha}\right)} \sum_{j=0}^{\infty}\left(\begin{array}{c}
1 / \alpha \\
j
\end{array}\right) \lambda\left(\frac{(s+\lambda)^{\alpha}}{\lambda^{\alpha}}-1\right)^{j} \\
& =\frac{1}{s}\left((s+\lambda)^{\alpha}-\lambda^{\alpha}\right) e^{-x\left((s+\lambda)^{\alpha}-\lambda^{\alpha}\right)} \lambda\left(\frac{(s+\lambda)^{\alpha}}{\lambda^{\alpha}}\right)^{1 / \alpha} \\
& =\left((s+\lambda)^{\alpha}-\lambda^{\alpha}\right) e^{-x\left((s+\lambda)^{\alpha}-\lambda^{\alpha}\right)}+\lambda \frac{1}{s}\left((s+\lambda)^{\alpha}-\lambda^{\alpha}\right) e^{-x\left((s+\lambda)^{\alpha}-\lambda^{\alpha}\right)} .
\end{aligned}
$$

Inverting the LT yields Eq. 29. 
Next, the asymptotic behavior of the moments of the ITS subordinator is discussed. Let $M_{q}(t)=\mathbb{E}\left(E_{\lambda, \alpha}(t)\right)^{q}, q>0$, be the $q$-th order moment of $E_{\lambda, \alpha}(t)$. Then the LT of $M_{q}(t)$ satisfies

$$
\begin{aligned}
\tilde{M}_{q}(s) & =\int_{0}^{\infty} e^{-s t} M_{q}(t) d t=-\int_{0}^{\infty} e^{-s t}\left(\int_{0}^{\infty} y^{q} \frac{d}{d y} \mathbb{P}\left(E_{\lambda, \alpha}(t)>y\right) d y\right) d t \\
& =q \int_{0}^{\infty} e^{-s t}\left(\int_{0}^{\infty} y^{q-1} \mathbb{P}\left(E_{\lambda, \alpha}(t)>y\right) d y\right) d t \\
& =q \int_{0}^{\infty} y^{q-1}\left(\int_{0}^{\infty} e^{-s t} \mathbb{P}\left(S_{\lambda, \alpha}(y) \leq t\right) d t\right) d y \\
& =\frac{q}{s} \int_{0}^{\infty} y^{q-1}\left(\int_{0}^{\infty} e^{-s t} f_{S_{\lambda, \alpha}(y)}(t) d t\right) d y \\
& =\frac{q}{s} \int_{0}^{\infty} y^{q-1} e^{-y \Psi_{S_{\lambda, \alpha}}(s)} d y=\frac{\Gamma(1+q)}{s\left(\Psi_{S_{\lambda, \alpha}}(s)\right)^{q}},
\end{aligned}
$$

where $\Psi_{S_{\lambda, \alpha}}(s)=(s+\lambda)^{\alpha}-\lambda^{\alpha}$.

An application of Tauberian Theorem (see e.g. Bertoin 1996, p.10), which relates the asymptotic behavior of a function to its Laplace transform, yields following asymptotic behavior of $M_{q}(t)$

$$
M_{q}(t) \sim\left\{\begin{array}{l}
\frac{\Gamma(1+q)}{\Gamma(1+q \alpha)} t^{q \alpha}, \quad \text { as } t \rightarrow 0 \\
\frac{\lambda^{q(1-\alpha)} t^{q}, \lambda>0, \quad \text { as } t \rightarrow \infty}{\Gamma^{q}} t^{q}, \lambda>0, \quad \text { as } t \rightarrow \infty \\
\frac{\Gamma(1+q)}{\Gamma(1+q \alpha)} t^{q \alpha}, \lambda=0 .
\end{array}\right.
$$

\section{FBM Delayed by Tempered Stable Subordinator}

In this section we introduce the process which arises after changing the time in a fractional Brownian motion by tempered stable subordinator. The new process is called subordinated FBM. First, the FBM and its basic properties are introduced to reuse them in later part of the paper. The FBM was introduced by Kolmogorov (Mandelbrot and Ness 1968; Kolmogoroff 1940) and very often is treated as an extension of the classical Brownian motion. Most of the properties of FBM are characterized by the self-similarity exponent $H$, called Hurst exponent. For any $0<H<1$ the FBM with index $H$ is a mean-zero Gaussian process $B_{H}(t)$ with the following representation (Mandelbrot and Ness 1968; Teuerle et al. 2013)

$$
B_{H}(t)=\int_{-\infty}^{\infty}\left((t-u)_{+}^{H-1 / 2}-(-u)_{+}^{H-1 / 2}\right) d B(u), \quad t \geq 0,
$$

where $B(t)$ is the Brownian motion and $(x)_{+}=\max (x, 0)$. It is worth mentioning, the process exhibits subdiffusive dynamics for $H<1 / 2$ and superdiffusive one for $H>1 / 2$. For each $t, \mathbb{E} B_{H}(t)=0, \mathbb{E} B_{H}^{2}(t)=t^{2 H}$ and its PDF is given by

$$
f_{B_{H}(t)}(x)=\frac{1}{\sqrt{2 \pi} t^{H}} e^{-\frac{1}{2} x^{2} t^{-2 H}}, x \in R .
$$

As it was mentioned, the FBM has $H$-self similarity property, which means for all $c>0$ the following holds

$$
\left\{B_{H}(c t), t \geq 0\right\} \stackrel{\mathcal{L}}{=}\left\{c^{H} B_{H}(t), t \geq 0\right\}
$$


One should remember that the fractional Gaussian noise, defined as the increments of FBM, $b_{H}(n)=B_{H}(n+1)-B_{H}(n)$ for $n=0,1, \ldots$, is time-correlated stationary process with covariance function

$$
\operatorname{Cov}\left(b_{H}(0), b_{H}(n)\right)=\mathbb{E}\left(b_{H}(0) b_{H}(n)\right)=\frac{1}{2}\left((n+1)^{2 H}+(n-1)^{2 H}-2 n^{2 H}\right) .
$$

Moreover, the Laplace transform of $B_{H}(t)$ is given by

$$
\mathbb{E}\left(e^{-u B_{H}(t)}\right)=e^{-\frac{1}{2} u^{2} t^{2 H}}, \quad t \geq 0 .
$$

The FBM time changed by the TSS $S_{\lambda, \alpha}(t)$ is defined by

$$
X(t)=B_{H}\left(S_{\lambda, \alpha}(t)\right),
$$

under the assumption that $B_{H}(t)$ and $S_{\lambda, \alpha}(t)$ are independent. In the following sections, the distributional properties of FBM time changed by the TSS are discussed. We establish the long-range dependence (LRD) of the process $X(t)$ and obtain the FPDE corresponding to its PDF.

\subsection{Asymptotic Moments}

It is well known that for a standard random variable $Z$ and for $q>0$ the following holds

$$
\mathbb{E}|Z|^{q}=\sqrt{\frac{2^{q}}{\pi}} \Gamma\left(\frac{1+q}{2}\right) \equiv c_{q} .
$$

For $0<q<1$, using independence of $B_{H}(t)$ and $S_{\lambda, \alpha}(t)$ and the self-similarity property of FBM, it follows,

$$
\begin{aligned}
\mathbb{E}|X(t)|^{q} & =\mathbb{E} S_{\lambda, \alpha}^{q H}(t) \mathbb{E}\left|B_{H}(1)\right|^{q} \\
& =c_{q} \mathbb{E} S_{\lambda, \alpha}^{q H}(t) \sim c_{q}\left(\alpha \lambda^{\alpha-1}\right)^{q H} t^{q H}, \text { as } t \rightarrow \infty,
\end{aligned}
$$

where constant $c_{q}$ is given in Eq. 36 .

\subsection{Long-Range-Dependence}

In this section we discuss the covariance and LRD behavior of process $X(t)$. Note that, a finite variance stationary process $Z(t)$ is said to have LRD property (Cont and Tankov 2003), if $\sum_{k=0}^{\infty} \gamma_{k}=\infty$, where

$$
\gamma_{k}=\operatorname{Cov}(Z(t), Z(t+k)) .
$$

In the following definition we give the equivalent definition for a non-stationary process $Z(t)$. 
Definition 1 Let $s>0$ be fixed and $t>s$. Then process $Z(t)$ is said to have LRD property if

$$
\operatorname{Corr}(\mathrm{Z}(\mathrm{s}), \mathrm{Z}(\mathrm{t})) \sim \mathrm{c}(\mathrm{s}) \mathrm{t}^{-\mathrm{d}}, \text { as } \mathrm{t} \rightarrow \infty
$$

where $c(s)$ is a constant depending on $s$ and $d \in(0,1)$.

The process $X(t)$ is not stationary and hence the definition 1 is used to establish the LRD. For fixed $s$ and large $t$, it follows

$$
\begin{aligned}
\mathbb{E}(X(t) X(s))= & \frac{1}{2}\left[\mathbb{E} X^{2}(t)+\mathbb{E} X^{2}(s)-\mathbb{E}(X(t)-X(s))^{2}\right] \\
= & \frac{1}{2}\left[\mathbb{E}\left(B_{H}\left(S_{\lambda, \alpha}(t)\right)\right)^{2}+\mathbb{E}\left(B_{H}\left(S_{\lambda, \alpha}(s)\right)\right)^{2}-\mathbb{E}\left(B_{H}\left(S_{\lambda, \alpha}(t-s)\right)\right)^{2}\right] \\
= & \frac{1}{2} \mathbb{E}\left(B_{H}(1)\right)^{2}\left[\mathbb{E}\left(S_{\lambda, \alpha}(t)\right)^{2 H}+\mathbb{E}\left(S_{\lambda, \alpha}(s)\right)^{2 H}-\mathbb{E}\left(S_{\lambda, \alpha}(t-s)\right)^{2 H}\right] \\
\approx & \frac{1}{2}\left[\left(\alpha \lambda^{\alpha-1}\right)^{2 H} t^{2 H}+\mathbb{E}\left(S_{\lambda, \alpha}(s)\right)^{2 H}-\left(\alpha \lambda^{\alpha-1}\right)^{2 H}(t-s)^{2 H}\right] \\
= & \frac{1}{2}\left(\alpha \lambda^{\alpha-1}\right)^{2 H} t^{2 H}\left(2 H \frac{s}{t}+\mathbb{E}\left(S_{\lambda, \alpha}(s)\right)^{2 H} t^{-2 H}+O\left(t^{-2}\right)\right) \\
& \sim H s\left(\alpha \lambda^{\alpha-1}\right)^{2 H} t^{2 H-1} .
\end{aligned}
$$

Using Eq. 37, it follows that

$$
\operatorname{Corr}(X(t), X(s)) \sim H s^{1-H} t^{H-1}, \text { as } t \rightarrow \infty .
$$

Thus the following proposition holds.

Proposition 5 The process $X(t)$ defined in Eq. 35 has LRD property for all $H \in(0,1)$ in sense of Definition 1.

\subsection{Partial Differential Equations Connection}

In this section the associated fractional-type partial differential equation for one dimensional PDF of the process (35) is obtained.

Proposition 6 The density $p(x, t)$ for $t>0$ of the process $X(t)$ defined in Eq. 35 satisfies the following equation

$$
\lambda p(x, t)-\left(\lambda^{\alpha}-\frac{\partial}{\partial t}\right)^{1 / \alpha} p(x, t)=H t^{2 H-1} \frac{\partial^{2}}{\partial x^{2}} p(x, t)
$$

with initial and boundary conditions

$$
\left\{\begin{array}{l}
p(x, 0)=\delta(x) \\
\lim _{|x| \rightarrow \infty} p(x, t)=0 \\
\lim _{|x| \rightarrow \infty} \frac{\partial}{\partial x} p(x, t)=0
\end{array}\right.
$$


Proof First let us observe that the initial and boundary conditions (41) are satisfied immediately since they hold for PDF of $B_{H}(t)$.

$$
\begin{aligned}
H t^{2 H-1} \frac{\partial^{2}}{\partial x^{2}} p(x, t) & =H t^{2 H-1} \frac{\partial^{2}}{\partial x^{2}} \int_{0}^{\infty} p_{H}^{n}(x, z) f_{\lambda, \alpha}(z, t) d z \\
& =\int_{0}^{\infty} \frac{\partial}{\partial z} p_{H}^{n}(x, z) f_{\lambda, \alpha}(z, t) d z \\
& =\left[p_{H}^{n}(x, z) f_{\lambda, \alpha}(z, t)\right]_{z=0}^{z=\infty}-\int_{0}^{\infty} p_{H}^{n}(x, z) \frac{\partial}{\partial z} f_{\lambda, \alpha}(z, t) d z \\
& =[\text { by the condition that } f(t, 0)=0 \text { for } t>0] \\
& =-\int_{0}^{\infty}\left(-\lambda f_{\lambda, \alpha}(z, t)-\left(\lambda^{\alpha}+\frac{\partial}{\partial t}\right)^{1 / \alpha} f_{\lambda, \alpha}(z, t)\right) d z \\
& =[\text { by Proposition } 2] \\
& =\lambda p(x, t)-\left(\lambda^{\alpha}-\frac{\partial}{\partial t}\right)^{1 / \alpha} p(x, t) .
\end{aligned}
$$

\section{FBM Delayed by Inverse Tempered Stable Subordinator}

Many real time-series exhibit constant time periods behavior i.e. the time-series remains at the same level for some intervals and also have characteristics similar to Gaussian processes although they are not Gaussian. Therefore there is need to consider new classes of systems to model these kinds of empirical behavior. Motivated by this fact, in this section we discuss the FBM time changed by the ITS subordinator $E_{\lambda, \alpha}(t)$, defined in Eq. 13. This process is defined by

$$
Y(t)=B_{H}\left(E_{\lambda, \alpha}(t)\right),
$$

where the processes $B_{H}(t)$ and $E_{\lambda, \alpha}(t)$ are assumed to be independent.

\subsection{Asymptotic Moments}

As in the previous case, we discuss the properties of the one-dimensional distribution of the process $Y(t)$ defined in Eq. 42. Using, Eqs. 30, 42 and self-similarity of FBM, it follows

$$
\begin{aligned}
\mathbb{E}|Y(t)|^{q} & =\mathbb{E} E_{\lambda, \alpha}^{q H}(t) \mathbb{E}\left|B_{H}(1)\right|^{q} \\
& \sim\left\{\begin{array}{l}
\frac{c_{q} \lambda^{q H(1-\alpha)}}{\alpha^{q} H} t^{q H}, \lambda>0, \\
\frac{c_{q} \Gamma(1+q H)}{\Gamma(1+q H \alpha)} t^{q H \alpha}, \lambda=0,
\end{array}\right.
\end{aligned}
$$

as $t \rightarrow \infty$, where $c_{q}$ is defined in Eq. 36 . 


\subsection{Long-Range-Dependence}

In this section we discuss the covariance and LRD behavior of process $Y(t)$ defined in Eq. 42. The covariance structure for time-changed FBM is discussed in Mijena (2014). An explicit asymptotic behavior for covariance structure of $Y(t)$ is provided here which helps in establishing the LRD property of the process $Y(t)$.

For $s<t$, the covariance function of $Y(t)$ is given by (see Theorem 3.1. Mijena 2014)

$$
\mathbb{E}(Y(s) Y(t))=M_{2 H}(s)+2 H \int_{0}^{s} M_{2 H-1}(t-y) d M_{1}(y),
$$

where $M_{q}(t)$ is the $q$-th order moment of $E_{\lambda, \alpha}(t)$. For fixed $s$ and large $t$, using Eq. 30, it follows

$$
\begin{aligned}
& \int_{0}^{s} M_{2 H-1}(t-y) d M_{1}(y) \\
& \sim \frac{\lambda^{(2 H-1)(1-\alpha)}}{\alpha^{(2 H-1)}} \int_{0}^{s}(t-y)^{2 H-1} M_{1}^{\prime}(y) d y \\
& \sim \frac{\lambda^{(2 H-1)(1-\alpha)}}{\alpha^{(2 H-1)}}(t-s)^{2 H-1} M_{1}(s) \\
& \sim \frac{\lambda^{(2 H-1)(1-\alpha)}}{\alpha^{(2 H-1)}} t^{2 H-1} M_{1}(s) .
\end{aligned}
$$

Thus $\mathbb{E}(Y(s) Y(t)) \sim M_{2 H}(s)+2 H \frac{\lambda^{(2 H-1)(1-\alpha)}}{\alpha^{(2 H-1)}} t^{2 H-1} M_{1}(s)$. For $\lambda>0$, using Eq. 43, it gives

$$
\operatorname{Corr}(Y(s), Y(t)) \sim \alpha^{2 H} s^{-H} \lambda^{-2 H(1-\alpha)} M_{2 H}(s) t^{-H}+2 H \lambda^{\alpha-1} \alpha^{2-2 H} s^{-H} M_{1}(s) t^{H-1},
$$

as $t \rightarrow \infty$. Hence, the following result holds.

Proposition 7 The process $Y(t)$ has the LRD property for all $H \in(0,1)$ in sense of definition 1.

\subsection{Partial Differential Equations Connection}

Similarly as in Proposition 6, an analogous version of associated shifted fractional partial differential equation for the PDF of the process $Y(t)$ defined in Eq. 42 is discussed here.

Proposition 8 The density $g(x, t)$ of the process $Y(t)$ defined in Eq. 42 satisfies the following equation

$$
\left(\lambda+\frac{\partial}{\partial t}\right)^{\alpha} g(x, t)-\lambda^{\alpha} g(x, t)+t^{-\alpha} M_{1,1-\alpha}^{1-\alpha}(-\lambda t) \delta(x)=H t^{2 H-1} \frac{\partial^{2}}{\partial x^{2}} g(x, t), t>0,
$$

with initial and boundary conditions

$$
\left\{\begin{array}{l}
g(x, 0)=\delta(x) \\
\lim _{|x| \rightarrow \infty} g(x, t)=0 \\
\lim _{|x| \rightarrow \infty} \frac{\partial}{\partial x} g(x, t)=0
\end{array}\right.
$$

Proof Again the conditions (45) are satisfied for the density of fractional Brownian motion $B_{H}(t)$, now the proof of Eq. 44 follows the same lines presented in Proposition 6. 


\section{Simulations}

In this section we discuss simulation procedures for both processes $X(t)$ and $Y(t)$ defined in the previous sections.

\subsection{FBM Delayed by Tempered Stable Subordinator}

The simulation procedures for sample paths (or trajectories) of subordinated processes have been widely described in the literature, for example FBM time-changed by gamma subordinator is discussed in Kozubowski et al. (2006) and FBM time-changed by Inverse Gaussian subordinator is analyzed in Wyłomańska et al. (2016).

The main idea is to simulate independent trajectories of the subordinator $S_{\lambda, \alpha}(t)$ and the FBM $B_{H}(t)$. By taking their superposition we obtain the trajectory of the process $X(t)$ defined in Eq. 35.

The procedure of simulation of FBM is described in details for example in Teuerle et al. (2013) therefore we refer the reader to this bibliography position.

In next step we simulate the trajectory of $S_{\lambda, \alpha}(t)$, that is a process of independent stationary increments having tempered stable distribution. The algorithm of simulation of tempered stable random variables has been described in Baeumer and Meerschaert (2010). We divide the interval $[0, T]$ into sub-intervals of length $\delta$ where the increments $S_{\lambda, \alpha}(t+\delta)-S_{\lambda, \alpha}(t), t=0, \delta, 2 \delta, \ldots, T-\delta$ have tempered stable distribution with Laplace transform $\mathbb{E}\left(e^{-u\left(S_{\lambda, \alpha}(t+\delta)-S_{\lambda, \alpha}(t)\right)}\right)=e^{-\delta\left((u+\lambda)^{\alpha}-\lambda^{\alpha}\right)}$. We simulate $[T / \delta]$ independent random variables from this distribution. Finally, the trajectory of $S_{\lambda, \alpha}(t)$ is obtained as the cumulative sum of the increments.

Finally, by taking superposition of trajectories of $B_{H}(t)$ and $S_{\lambda, \alpha}(t)$ we obtain approximated trajectory of process $X(t)$. The exemplary trajectories of the process $X(t)$ obtained by using the described procedure are presented in Fig. 1.
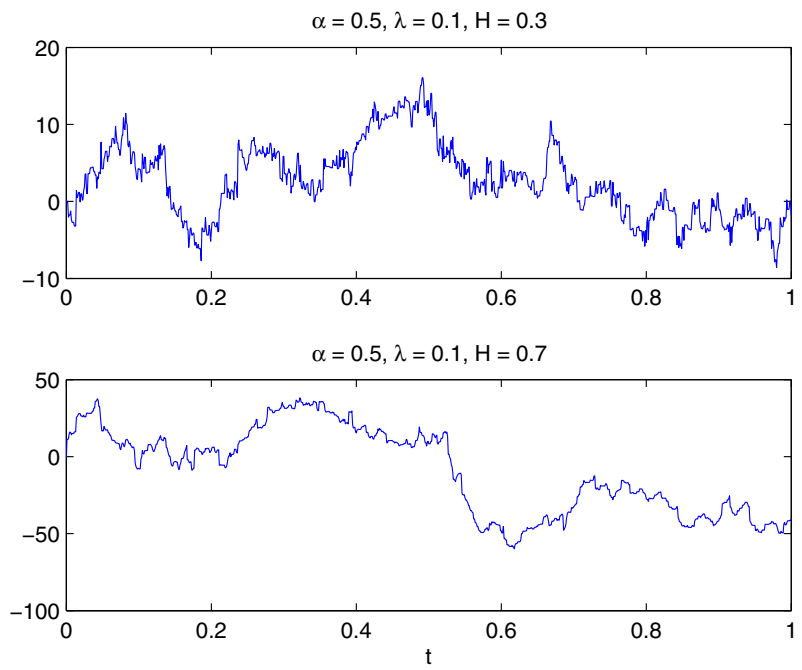

Fig. 1 The exemplary trajectories of the process $X(t)$ for $H=0.3$ (top panel) and $H=0.7$ (bottom panel) 



Fig. 2 The exemplary trajectories of the process $Y(t)$ for $H=0.3$ (top panel) and $H=0.7$ (bottom panel)

\subsection{FBM Delayed by Inverse Tempered Stable Subordinator}

The idea of simulation of $Y(t)$ process is similar as presented above. It is also based on the simulation of independent trajectories of FBM and the process $E_{\lambda, \alpha}(t)$ which is an inverse to the tempered stable process $S_{\lambda, \alpha}(t)$. In order to simulate the approximate trajectory of the inverse tempered stable subordinator first we need to define $E_{\lambda, \alpha}$ with the step length $\delta$ in the following way

$$
E_{\lambda, \alpha, \delta}(t)=\left(\min \left\{n \in \mathbb{N}: S_{\lambda, \alpha}(\delta n)>t\right\}-1\right) \delta, \quad n=1,2, \ldots,
$$

where $S_{\lambda, \alpha}(\delta n)$ is the value of tempered stable subordinator $S_{\lambda, \alpha}(t)$ evaluated at $\delta n$, which can be simulated by using the method presented above. Observe that trajectory $E_{\lambda, \alpha}(t)$ has increments of length $\delta$ at random time instants governed by process $S_{\lambda, \alpha}(\tau)$ and therefore $E_{\lambda, \alpha, \delta}(t)$ is approximation of operational time. Finally, the trajectory of the process $Y(t)$ is obtained as the superposition of FBM, for which the simulation procedure is mentioned above, and the process $E_{\lambda, \alpha}(t)$. The exemplary trajectories of the process $Y(t)$ obtained by using described procedure are presented in Fig. 2. We observe here the constant time periods characteristic for processes delayed by inverse subordinators.

\section{Conclusions}

In this paper we have considered the FBM delayed by tempered stable and inverse tempered stable subordinators. The tempered stable process in the literature is considered as the extension of the popular stable process. The main advantage of the tempered stable systems is that they posses many properties of stable systems however they have all finite moments therefore the classical methods of their analysis can be applied. The subordinated processes are especially useful in modeling of anomalous diffusion phenomena. In this paper we have compared the main properties of time-changed FBM. The special attention 
is paid to long-range dependence property and form of fractional partial differential equation for probability density function of analyzed systems. The theoretical part of the paper is enriched by simulation procedures description.

Acknowledgments This work is supported by National Center of Science Opus Grant No. 2016/21/B/ST1/ 00929 "Anomalous diffusion processes and their applications in real data modelling".

Open Access This article is distributed under the terms of the Creative Commons Attribution 4.0 International License (http://creativecommons.org/licenses/by/4.0/), which permits unrestricted use, distribution, and reproduction in any medium, provided you give appropriate credit to the original author(s) and the source, provide a link to the Creative Commons license, and indicate if changes were made.

\section{Appendix A}

\section{Laplace-Erdelyi Theorem}

Consider the integral

$$
I(t)=\int_{a}^{b} e^{-t f(x)} g(x) d x,
$$

where $(a, b)$ is a real (finite or infinite) interval. Further, $t$ is large positive and the functions $f$ and $g$ are continuous. Suppose $f$ have a single minima at $x=a$ and functions $f$ and $g$ are such that

$$
f(x) \sim f(a)+\sum_{j=0}^{\infty} a_{j}(x-a)^{j+\beta}, \beta>0
$$

and

$$
g(x) \sim \sum_{j=0}^{\infty} b_{j}(x-a)^{j+\gamma-1}, \gamma>0 .
$$

Then, the asymptotic expansion of the integral $I(t)$ is given by

$$
I(t) \sim e^{-t f(a)} \sum_{n=0}^{\infty} \Gamma\left(\frac{n+\gamma}{\beta}\right) \frac{c_{n}}{t^{(n+\gamma) / \beta}},
$$

where $c_{n}$ is given by

$$
c_{n}=\frac{1}{a_{0}^{(n+\gamma) / \beta}} \sum_{j=0}^{n} b_{n-j} \sum_{i=0}^{j}\left(\begin{array}{c}
-\frac{n+\gamma}{\beta} \\
i
\end{array}\right) \frac{1}{a_{0}^{i}} \hat{B}_{j, i}\left(a_{1}, a_{2}, \cdots, a_{j-i+1}\right),
$$

where $\hat{B}_{j, i}$ are the partial (or incomplete) ordinary Bell polynomials (Andrews 1998).

\section{Partial Exponential Bell Polynomials}

The partial (or incomplete) exponential Bell polynomials are a triangular array of polynomials given by

$$
B_{n, k}\left(x_{1}, x_{2}, \ldots, x_{n-k+1}\right)=\sum \frac{n !}{j_{1} ! j_{2} ! \cdots j_{n-k+1} !}\left(\frac{x_{1}}{1 !}\right)^{j_{1}}\left(\frac{x_{2}}{2 !}\right)^{j_{2}} \cdots\left(\frac{x_{n-k+1}}{(n-k+1) !}\right)^{j_{n-k+1}} \text {, }
$$

where the sum is taken over the sequences satisfying

$$
j_{1}+j_{2}+\cdots+j_{n-k+1}=k, j_{1}+2 j_{2}+3 j_{3}+\cdots+(n-k+1) j_{n-k+1}=n,
$$


where $j_{1}, j_{2}, j_{3}, \ldots, j_{n-k+1}$ are non-negative integers. Further, the sum

$$
B_{n}\left(x_{1}, \ldots, x_{n}\right)=\sum_{k=1}^{n} B_{n, k}\left(x_{1}, x_{2}, \ldots, x_{n-k+1}\right)
$$

is called the $n$-th complete exponential Bell polynomial.

\section{Partial Ordinary Bell Polynomial}

The partial ordinary Bell polynomial is given by

$$
\hat{B}_{n, k}\left(x_{1}, x_{2}, \ldots, x_{n-k+1}\right)=\sum \frac{k !}{j_{1} ! j_{2} ! \cdots j_{n-k+1} !} x_{1}^{j_{1}} x_{2}^{j_{2}} \cdots x_{n-k+1}^{j_{n-k+1}},
$$

where the sum runs over all sequences $j_{1}, j_{2}, j_{3}, \cdots, j_{n-k+1}$ of non-negative integers such that

$$
j_{1}+j_{2}+\cdots+j_{n-k+1}=k, j_{1}+2 j_{2}+\cdots+(n-k+1) j_{n-k+1}=n .
$$

\section{References}

Abramowitz M, Stegun IA (1972) Handbook of mathematical functions: with formulas, graphs, and mathematical tables. Dover books on mathematics. Dover, New York

Andrews GE (1998) The theory of partitions. Cambridge Mathematical Library. Cambridge University Press, Cambridge

Applebaum D (2009) Lévy processes and stochastic calculus. Cambridge University Press (CUP), Cambridge. https://doi.org/10.1017/cbo9780511809781

Baeumer B, Meerschaert MM (2010) Tempered stable lévy motion and transient super-diffusion. J Comput Appl Math 233(10):2438-2448. https://doi.org/10.1016/j.cam.2009.10.027

Barndorff-Nielsen OE, Pedersen J, Sato K-I (2001) Multivariate subordination, self-decomposability and stability. Adv Appl Probab 33(1):160-187. https://doi.org/10.1239/aap/999187902

Beghin L (2015) On fractional tempered stable processes and their governing differential equations. J Comput Phys 293:29-39. https://doi.org/10.1016/j.jcp.2014.05.026

Bertoin J (1996) Lévy processes (Cambridge tracts in mathematics), 1st edn. Cambridge University Press, Cambridge

Bochner S (1949) Diffusion equation and stochastic processes. Proc Natl Acad Sci USA 35(7):368-370

Bochner S (1955) Harmonic analysis and the theory of probability. University of California Press, Berkeley

Clark P (1973) A subordinated stochastic process model with finite variance for speculative prices. Econometrica 41(1):135-55

Cont R, Tankov P (2003) Financial modelling with jump processes. Chapman and Hall/CRC, London. https://doi.org/10.1201/9780203485217

Doukhan P, Oppenheim G, Taqqu MS (eds) (2003) Theory and applications of long-range dependence. Birkhäuser Boston Inc., Boston

Fristedt B (1977) Local behavior of stable subordinators. Adv Appl Probab 9(2):194-195. https://doi.org/ $10.1017 /$ S0001867800043366

Gorenflo R, Mainardi F (1997) Fractional calculus. Springer Vienna, Vienna, pp 223-276. https://doi.org/ 10.1007/978-3-7091-2664-6_5

Kolmogoroff AN (1940) Wienersche Spiralen und einige andere interessante Kurven im Hilbertschen Raum. C R (Doklady) Acad Sci URSS (N.S.) 26:115-118

Kozubowski TJ, Meerschaert MM, Podgórski K (2006) Fractional laplace motion. Adv Appl Probab 38(2):451-464. https://doi.org/10.1239/aap/1151337079

Kumar A, Vellaisamy P (2015) Inverse tempered stable subordinators. Stat Probab Lett 103:134-141. https://doi.org/10.1016/j.spl.2015.04.010

Magdziarz M (2009) Langevin picture of subdiffusion with infinitely divisible waiting times. J Stat Phys 135(4):763-772. https://doi.org/10.1007/s10955-009-9751-z

Mandelbrot BB, Ness JWV (1968) Fractional brownian motions, fractional noises and applications. SIAM Rev 10(4):422-437. https://doi.org/10.1137/1010093 
Meerschaert MM, Scheffler H-P (2008) Triangular array limits for continuous time random walks. Stoch Process Appl 118(9):1606-1633. https://doi.org/10.1016/j.spa.2007.10.005

Meerschaert MM, Straka P (2013) Inverse stable subordinators. Math Model Nat Phenom 8(2):1-16. https://doi.org/10.1051/mmnp/20138201

Mijena JB (2014) Correlation structure of time-changed fractional brownian motion, arXiv:1408.4502

Monje CA, Chen Y, Vinagre BV, Xue D, Feliu-Batlle V (2010) Fractional-order systems and controls: fundamentals and applications. Springer, London

Nezhadhaghighi MG, Rajabpour MA, Rouhani S (2011) First-passage-time processes and subordinated Schramm-Loewner evolution. Phys Rev E 84:011134. https://doi.org/10.1103/PhysRevE.84.011134

Prabhakar TR (1971) A singular integral equation with a generalized mittag-leffler function in the kernel. Yokohama Math J 19:7-15

Rosiński J (2007) Tempering stable processes. Stoch Process Appl 117(6):677-707. https://doi.org/10. 1016/j.spa.2006.10.003

Rota G-C, Shen J (2000) On the combinatorics of cumulants. J Comb Theory Ser A 91(1):283-304. https://doi.org/10.1006/jcta.1999.3017

Samorodnitsky G, Taqqu MS (1994) Levy measures of infinitely divisible random vectors and slepian inequalities. Ann Probab 22(4):1930-1956. https://doi.org/10.1214/aop/1176988490

Sato K-I (1999) Lévy processes and infinitely divisible distributions. Cambridge University Press, Cambridge

Scher H, Margolin G, Metzler R, Klafter J, Berkowitz B (2002) The dynamical foundation of fractal stream chemistry: the origin of extremely long retention times. Geophys Res Lett 29(5):5-1-5-4. https://doi.org/10.1029/2001GL014123

Smith PJ (1995) A recursive formulation of the old problem of obtaining moments from cumulants and vice versa. Am Stat 49(2):217-218. https://doi.org/10.1080/00031305.1995.10476146

Stanislavsky A, Weron K, Weron A (2014) Anomalous diffusion with transient subordinators: a link to compound relaxation laws. J Chem Phys 140(5):054113. https://doi.org/10.1063/1.4863995

Teuerle M, Wyłomańska A, Sikora G (2013) Modeling anomalous diffusion by a subordinated fractional Lévy-stable process. J Stat Mech: Theory Exp 2013(05):P05016. https://doi.org/10.1088/1742$5468 / 2013 / 05 / \mathrm{p} 05016$

Wyłomańska A (2013) The tempered stable process with infinitely divisible inverse subordinators. J Stat Mech: Theory Exp 2013(10):P10011

Wyłomańska A, Kumar A, Połoczański R, Vellaisamy P (2016) Inverse gaussian and its inverse process as the subordinators of fractional Brownian motion. Phys Rev E 94:042128. https://doi.org/10.1103/ PhysRevE.94.042128 